\title{
Produção e heterose de híbridos experimentais de pepino do tipo japonês ${ }^{1}$
}

\author{
Ariane Teixeira da Silva Lima² \& Antonio Ismael Inácio Cardoso ${ }^{3}$
}

\section{RESUMO}

A utilização de híbridos $\mathrm{F}_{1}$ é motivada pelas vantagens oferecidas aos produtores, destacando-se o aumento da produtividade. O objetivo deste trabalho foi avaliar o potencial produtivo de 12 linhagens endogâmicas $\left(\mathrm{S}_{5}\right)$, obtidas a partir de autofecundações sucessivas de duas populações de pepino japonês: população RY (Rensei x Yoshinari) e população TT (Tsuyataro x Taisho) e de 18 híbridos experimentais, obtidos a partir do cruzamento entre estas linhagens no esquema de dialelo parcial circulante interpopulacional. O experimento foi conduzido de janeiro a abril de 2008 , no delineamento de blocos ao acaso, com 33 tratamentos (18 híbridos experimentais, 12 linhagens e três híbridos comerciais: Tsuyataro, Taisho e Yoshinari), com quatro repetições e cinco plantas por parcela. Foram avaliadas as seguintes características: produção de frutos por planta, total e comercial, percentagem (\%) de frutos comerciais, massa média de frutos comerciais, comprimento, diâmetro e relação entre comprimento e diâmetro (C/D) dos frutos. As médias foram agrupadas pelo teste de Scott Knott (5\%). A linhagem (TT2) e seis híbridos experimentais (H16, H26, H11, H43, H54 e H15) foram tão ou mais produtivos que o melhor híbrido comercial, Tsuyataro. No geral, as heteroses para produção de frutos foram positivas e elevadas, enquanto, para as características de fruto (comprimento, diâmetro, relação C/D e massa média) foram de menor valor.

Palavras-chave: Cucumis sativus, melhoramento, produção, híbridos.

\section{ABSTRACT}

\section{Production and heterosis of experimental hybrids of Japanese cucumber}

Utilization of $\mathrm{F}_{1}$ hybrids is motivated due to the advantages to producers, particularly yielding increases. The objective of this work was to evaluate the potential yield of 12 inbred lines $\left(\mathrm{S}_{5}\right)$ of two populations of Japanese cucumber: population RY $=($ Rensey $x$ Yoshinari $)$ and population $\mathrm{TT}=($ Tsuyataro $\mathrm{x}$ Taisho $)$ and 18 experimental hybrids obtained from a circular partial interpopulation diallel. The experiment was carried out from January to April, 2008. The treatments were arranged in a randomized blocks design, with 33 treatments (18 experimental hybrids, 12 lines and three commercials hybrids: Tsuyataro, Taisho and Yoshinari), with four replications and five plants per plot. Fruit yield per plant, total and marketable; percentage (\%) of marketable fruits; average mass of marketable fruits; length, diameter and relation length/diameter (L/D) of fruits were evaluated. Treatment means were compared by the Scott Knott test (5\%). Line (TT2) and six experimental hybrids (H16, H26, H11, H43, H54 and H15) were as productive as, or more than, the best commercial hybrid, Tsuyataro. In general, the heterosis for fruit yield was positive and high, while heterosis for fruit characteristics (length, diameter, relation L/D and average fruit weight) was of smaller values.

Key words: Cucumis sativus, breeding, yield, hybrids.

\footnotetext{
Recebido para publicação em 10/06/2011 e aprovado em 04/07/2012.

${ }^{1}$ Parte da dissertação da primeira autora. Bolsista Fapesp.

2 Engenheira-Agrônoma, Mestre. Departamento de Produção Vegetal, Universidade Estadual Paulista “Júlio de Mesquita Filho”, Caixa Postal 237, 18610-307, Botucatu, São Paulo, Brasil. ariane_lima@hotmail.com

${ }^{3}$ Engenheiro-Agrônomo, Doutor. Departamento de Produção Vegetal, Universidade Estadual Paulista "Júlio de Mesquita Filho", Caixa Postal 237, 18610-307, Botucatu, São Paulo, Brasil. ismaeldh@fca.unesp.br (autor correspondente).
} 


\section{INTRODUÇÃO}

O pepineiro (Cucumis sativus L.) é uma espécie não adaptada ao cultivo sob baixas temperaturas, sendo o desenvolvimento da planta favorecido por temperaturas superiores a $20^{\circ} \mathrm{C}$, pois temperaturas inferiores a esta afetam a absorção de água e nutrientes pelo sistema radicular (Robinson \& Decker-Walters, 1999). Esta baixa adaptação ao cultivo sob baixas temperaturas foi um dos motivos pelos quais os produtores brasileiros passaram a cultivar pepino em ambiente protegido, a partir da década de 1980, sendo uma das hortaliças mais cultivadas sob ambiente protegido (Cañizares, 1998; Cardoso \& Wilcken, 2008).

No Estado de São Paulo, Brasil, os híbridos do tipo japonês são os mais cultivados e têm a preferência dos consumidores (Filgueira, 2003). Entretanto, quase todos os híbridos de pepino japonês utilizados no Brasil são importados, representando altos gastos anuais em importação de sementes. A maioria dos híbridos de pepino tipo japonês são partenocárpicos, podendo ser cultivados o ano todo em ambiente protegido, mesmo em ambientes fechados (Filgueira, 2003; Cardoso \& Silva, 2003).

A superioridade de híbridos $F_{1}$ de pepino foi constatada há mais de nove décadas, em 1916, por Hayes \& Jones, citados por Filgueira et al. (1986). Nessa ocasião, verificou-se a presença de heterose para produção de frutos e número de frutos por planta. Entretanto, o primeiro híbrido comercial de pepino, 'Burpee Hybrid', foi obtido por O. Shifriss, em 1945, e introduzido nos E.U.A. pela "Burpee Seed Company" (Robinson \& Decker-Walters, 1999).

Vários autores têm relatado heterose em pepino (Ghaderi \& Lower, 1978; Filgueira et al., 1986; Rubino \& Wehner, 1986; Delaney \& Lower, 1987; Cardoso, 2006, 2007; Godoy et al., 2008), o que pode justificar a adoção de híbridos $\mathrm{F}_{1}$.

Por isso, a utilização de híbridos $\mathrm{F}_{1}$ é motivada pelas vantagens oferecidas aos produtores e consumidores, destacando-se o aumento da produtividade, precocidade, maior uniformidade, melhor padronização e qualidade dos frutos, maior resistência a pragas e doenças e estabilidade de comportamento sob condições ambientais variáveis (Maluf, 2001).

A obtenção de híbridos experimentais pode ser feita a partir de cruzamentos entre diversas linhagens, em um esquema denominado de cruzamentos dialélicos, ou seja, quando se realizam todos os cruzamentos possíveis entre um conjunto de n linhagens (Miranda Filho \& Gorgulho, 2001). O sistema de cruzamentos dialélicos mostra-se bastante eficiente para avaliar as linhagens, pois, além de identificar os melhores híbridos simples, também auxilia na seleção daquelas mais promissoras para entrarem em um programa de melhoramento.

Dialelos completos têm-se limitado a número restrito de genitores, pois, com o aumento destes, ocorre eleva- ção no número de cruzamentos a serem realizados, associada às dificuldades de sua avaliação em campo com a precisão experimental desejada, tornando inviável a avaliação de todos os cruzamentos possíveis (Miranda Filho \& Gorgulho, 2001). Por isso o sistema dialelo circulante é um tipo no qual o estudo é realizado por meio da análise de uma amostra de todos os possíveis cruzamentos entre os genitores, ao contrário do sistema dos dialelos completos, em que todas as combinações entre genitores são incluídas (Cruz \& Regazzi, 2004).

Este trabalho teve como objetivo avaliar o potencial produtivo de híbridos $\mathrm{F}_{1}$ obtidos em cruzamentos dialélicos parciais circulantes interpopulacionais entre 12 linhagens endogâmicas de pepino japonês, assim como estimar a heterose dos híbridos experimentais.

\section{MATERIAL E MÉTODOS}

Todas as etapas foram conduzidas na Fazenda Experimental São Manuel, localizada no município de São Manuel (SP), pertencente à Faculdade de Ciências Agronômicas (FCA) da Universidade Estadual Paulista (UNESP), Campus de Botucatu. A localidade apresenta as seguintes coordenadas geográficas: longitude $48^{\circ} 34^{\prime} \mathrm{W}$, latitude $22^{\circ} 44^{\prime} \mathrm{S}$ e altitude média de 750 metros. O experimento foi conduzido em cultivo protegido, utilizando-se estruturas não climatizadas, tipo arco, com cerca de $7 \mathrm{~m}$ de largura por $20 \mathrm{~m}$ de comprimento e pé-direito de $1,8 \mathrm{~m}$, com cobertura de polietileno transparente de $150 \mu \mathrm{m}$ de espessura.

Foram avaliados 33 tratamentos: 18 híbridos experimentais, 12 linhagens e três híbridos comerciais (Tsuyataro, Taisho e Yoshinari), no delineamento em blocos ao acaso, com quatro repetições e cinco plantas por parcela.

As 12 linhagens $\mathrm{S}_{5}$ foram amostradas ao acaso, seis de cada população: população RY (Rensei x Yoshinari) e população TT (Tsuyataro x Taisho). Estas populações foram obtidas a partir do estudo de Cardoso \& Silva (2003), que identificaram os híbridos Tsuyataro, Yoshinari, Taisho e Rensei como aqueles com melhor adaptação, produção e qualidade de frutos para as condições de São ManuelSP. Estes foram intercruzados e foram realizadas cinco gerações de autofecundação, conduzidas pelo método de "Single Seed Descent", até a obtenção das linhagens $\mathrm{S}_{5}$ de cada população.

Os 18 híbridos experimentais foram obtidos pelo cruzamento entre linhagens de populações diferentes (interpopulacional), sendo que cada uma das seis linhagens da população RY foi cruzada com apenas três linhagens da população TT, caracterizando o modelo dialélico parcial circulante - interpopulacional com o mesmo número de cruzamentos para cada linhagem (três), segundo o modelo sugerido por Miranda Filho \& Gorgulho (2001). 
Todos os cruzamentos foram efetuados conforme recomendação de Lower \& Edwards (1986). As pontas das pétalas das flores masculinas e femininas selecionadas, ainda fechadas, foram amarradas com lã na tarde anterior ao cruzamento. A polinização foi feita na manhã seguinte, encostando-se as anteras nos estigmas das flores femininas, deixando todo o pólen aderido. Após a polinização, as flores femininas foram protegidas por um saco de papel e um clipe que o prendia ao pedúnculo (evitando a polinização por insetos). Também no pedúnculo, colocava-se uma etiqueta, identificando o cruzamento (fonte do pólen) e a data. As flores femininas que não foram aproveitadas nos cruzamentos foram eliminadas, para que não houvesse competição com os frutos provenientes dos cruzamentos.

A semeadura foi realizada em 22/01/2008, em bandejas de poliestireno expandido de 128 células. Em 08/02/2008, quando as mudas apresentavam três folhas verdadeiras, ocorreu o transplante, para vasos de 15 litros, que continham o substrato comercial Rendmax ${ }^{\circledR}$, para evitar problemas com patógenos de solo. O espaçamento foi de 1,0 $\mathrm{m}$ entre linhas e $0,5 \mathrm{~m}$ entre plantas (centro a centro dos vasos).

Cada planta foi tutorada individualmente, eliminandose todas as brotações e flores até o $5^{\circ}$ nó da haste principal, fez-se a desbrota das ramas laterais (após o $6^{\circ}$ nó da haste principal), entre a segunda e terceira folhas, e foi retirado o meristema apical da planta ao atingir a altura do arame (cerca de 1,80 m de altura).

Foram realizadas três colheitas, por semana, de 14/03/ 2008 a 21/04/2008. Os frutos foram colhidos imaturos, com cerca de $20 \mathrm{~cm}$ de comprimento e, posteriormente, pesados em balança de precisão e classificados como frutos comerciais (sem defeitos aparentes e retos) ou não comerciais.

Foram avaliadas as seguintes características: produção de frutos (número e massa por planta) total e comercial, percentagem $(\%)$ de frutos comerciais e massa média de frutos comerciais. Em uma amostra de dez frutos por parcela, foram avaliados o comprimento $(\mathrm{C})$, o diâmetro (D) e a relação entre eles (C/D), para caracterização dos frutos.

Foram feitas as análises de variância para cada característica avaliada e as médias dos 33 tratamentos foram agrupadas pelo teste de Scott Knott $(\mathrm{p}<0,05)$, para verificar o potencial dos híbridos experimentais, comparativamente aos comerciais. Para as características número de frutos total e comercial foi realizada transformação dos dados em $\sqrt{ }$, enquanto, para percentagem de frutos comerciais, a transformação foi em $\operatorname{arcsen} \sqrt{ }$, antes de se realizarem as análises estatísticas. Porém, nas tabelas constam os valores sem as transformações. Também foram estimadas as heteroses $(\%)$ dos híbridos experimentais em relação às médias dos genitores.

\section{RESULTADOS E DISCUSSÃO}

Para a característica número de frutos total por planta (FT), todas as linhagens da população TT (Tsuyataro x Taisho) foram superiores às linhagens da população RY (Rensei x Yoshinari) e a dois híbridos comerciais (Taisho e Yoshinari) e não diferiram do melhor híbrido comercial, Tsuyataro (Tabela 1). 61\% dos híbridos experimentais não diferiram do híbrido Tsuyataro e foram superiores aos outros dois híbridos comerciais.

Para massa de frutos total por planta (MT), os híbridos experimentais apresentaram comportamento semelhante ao observado para número de frutos total por planta, exceto o H55, que foi inferior ao híbrido comercial Tsuyataro. Porém, para as linhagens da população TT, apenas TT2 e TT6 foram semelhantes ao melhor híbrido comercial, Tsuyataro (Tabela 1). A produção em massa, considerando-se, neste trabalho, 2 plantas $\mathrm{m}^{-2}$ (espaçamento de 1,0 x 0,5 m), variou de 3,11 a $6,42 \mathrm{~kg} \mathrm{~m}^{-2}$, resultado inferior aos encontrados por Cardoso \& Silva (2003), que variaram de 7,45 a 11,18 $\mathrm{kg} \mathrm{m}^{-2}$, e superior aos obtidos por Machado et al. (2001), que, para pepino japonês, obtiveram valores de produção variando de 0,154 a $0,606 \mathrm{~kg} \mathrm{~m}^{-2}$.

Para número de frutos comerciais por planta (FC), as linhagens TT2 e TT3 e os híbridos experimentais H16, H26, H11, H22, H43, H54, H15 e H65 e o híbrido Tsuyataro não apresentaram diferença estatística entre si, sendo superiores aos híbridos Taisho e Yoshinari (Tabela 1). Quanto ao híbrido experimental H65, apesar de ele não ser muito produtivo, a maioria de seus frutos $(51 \%)$ possui boas características comerciais, ao contrário, por exemplo, dos híbridos experimentais H66, H31 e H44, que, apesar de produtivos, possuem, em média, $70 \%$ dos frutos não comerciais.

Os resultados obtidos para massa de frutos comerciais (MC) são ligeiramente diferentes da característica anterior, pois a maioria dos híbridos experimentais (15 de 18) e todos os comerciais estão no grupo superior, além das linhagens RY1, RY5, TT2 e TT3. Considerando-se que a comercialização dos frutos normalmente é feita por massa, a maioria dos híbridos experimentais equivaleu aos híbridos comerciais (Tabela 1).

Os maiores valores nos coeficientes de variação foram obtidos para as características massa de frutos totais $(26,8 \%)$ e comerciais $(31,4 \%)$ (Tabela 1$)$, provavelmente pelo fato de as colheitas não serem realizadas diariamente e o crescimento dos frutos ser muito rápido. Desta maneira, na maioria das colheitas, havia frutos pouco passados do ponto ideal, afetando o valor da massa de frutos de maneira desigual entre as parcelas. Esta mesma tendência foi observada em outras pesquisas com a cultura, por Cardoso (2002), Godoy et al. (2005) e Oviedo et al. (2008). 
A percentagem de frutos comerciais variou de 27,6 a 55,2\% (Tabela 1), havendo diferença estatística significativa entre os tratamentos. Em média, 59\% dos frutos apresentaram algum tipo de defeito. Esta é uma taxa alta, porém, não foram realizados desbastes de frutos defeituosos, mesmo quando estes já apresentavam defeitos no início do desenvolvimento. Com o desbaste de frutos defeituosos, há um estímulo para o desenvolvimento de novos frutos de melhor qualidade (Nomura $\&$ Cardoso, 2000). Além disto, foram considerados comerciais apenas frutos sem quaisquer tipos de defeitos, ou seja, frutos que seriam comercializados como os de melhor qualidade. Entre os defeituosos foram in- cluídos frutos um pouco tortos que poderiam ser comercializados por um preço menor. Porém, preferiuse considerar comerciais apenas os frutos totalmente isentos de defeitos aparentes que poderiam reduzir seu preço na comercialização, resultando em elevada taxa de frutos não comerciais. Estes dados são muito inferiores aos relatados por Cardoso (2007), que obteve, em média, $20 \%$ de frutos defeituosos. Os híbridos comerciais não apresentaram diferença estatística entre si. As linhagens TT2 e TT3 e os híbridos experimentais H22, H15 e H65 apresentaram a maior percentagem de frutos comerciais, sendo superiores aos híbridos comerciais.

Tabela 1. Médias do número de frutos total por planta (FT), massa de frutos total por planta (MT), número de frutos comerciais por planta (FC), massa de frutos comerciais por planta (MC) e percentagem de frutos comerciais (\%FC)

\begin{tabular}{|c|c|c|c|c|c|}
\hline Linhagem ou híbrido & $\begin{array}{c}\text { FT } \\
\left(\mathbf{n}^{0} \text { planta }^{-1}\right)\end{array}$ & $\begin{array}{c}\text { MT } \\
\left(\text { g planta }^{-1}\right)\end{array}$ & $\begin{array}{c}\text { FC } \\
\left(\mathbf{n}^{0} \text { planta }^{-1}\right)\end{array}$ & $\begin{array}{c}\text { MC } \\
\left(\text { g planta }^{-1}\right)\end{array}$ & $\begin{array}{c}\% \mathrm{FC} \\
\%\end{array}$ \\
\hline RY1 & $21,25 \mathrm{~b}$ & $1627 \mathrm{~b}$ & $9,00 \mathrm{~b}$ & $1075 \mathrm{a}$ & $41,7 \mathrm{~b}$ \\
\hline RY2 & $13,25 \mathrm{~b}$ & $1558 \mathrm{~b}$ & $4,75 \mathrm{~b}$ & $550 \mathrm{~b}$ & $35,1 \mathrm{c}$ \\
\hline RY3 & $14,50 \mathrm{~b}$ & $1679 \mathrm{~b}$ & $4,75 \mathrm{~b}$ & $587 \mathrm{~b}$ & $33,3 \mathrm{c}$ \\
\hline RY4 & $20,00 \mathrm{~b}$ & $1919 \mathrm{~b}$ & $7,25 \mathrm{~b}$ & $784 \mathrm{~b}$ & $35,2 \mathrm{c}$ \\
\hline RY5 & $17,25 \mathrm{~b}$ & $1962 \mathrm{~b}$ & $7,50 \mathrm{~b}$ & $905 \mathrm{a}$ & $41,8 \mathrm{~b}$ \\
\hline RY6 & $18,75 \mathrm{~b}$ & $1825 \mathrm{~b}$ & $8,00 \mathrm{~b}$ & $737 \mathrm{~b}$ & $43,2 \mathrm{~b}$ \\
\hline TT1 & $23,00 \mathrm{a}$ & $2343 \mathrm{~b}$ & $6,25 \mathrm{~b}$ & $658 \mathrm{~b}$ & $27,6 \mathrm{c}$ \\
\hline TT2 & $24,25 \mathrm{a}$ & $2431 \mathrm{a}$ & $13,25 \mathrm{a}$ & $1421 \mathrm{a}$ & $55,2 \mathrm{a}$ \\
\hline TT3 & $23,25 \mathrm{a}$ & $2115 b$ & $12,25 \mathrm{a}$ & $1336 \mathrm{a}$ & $52,4 \mathrm{a}$ \\
\hline TT4 & $22,75 \mathrm{a}$ & $2166 b$ & $9,25 \mathrm{~b}$ & $990 \mathrm{~b}$ & $39,1 \mathrm{c}$ \\
\hline TT5 & $23,00 \mathrm{a}$ & $2198 b$ & $7,25 \mathrm{~b}$ & $789 \mathrm{~b}$ & $33,1 \mathrm{c}$ \\
\hline TT6 & $25,25 \mathrm{a}$ & $3053 \mathrm{a}$ & $7,00 \mathrm{~b}$ & $756 \mathrm{~b}$ & $28,8 \mathrm{c}$ \\
\hline H16 (RY1 x TT6) & $23,50 \mathrm{a}$ & $2617 \mathrm{a}$ & $10,75 \mathrm{a}$ & $1237 \mathrm{a}$ & $45,9 \mathrm{~b}$ \\
\hline H26 (RY2 x TT6) & $25,25 \mathrm{a}$ & $2746 \mathrm{a}$ & $11,50 \mathrm{a}$ & $1292 \mathrm{a}$ & $44,8 \mathrm{~b}$ \\
\hline H66 (RY6 x TT6) & $22,50 \mathrm{a}$ & 2527 a & $7,50 \mathrm{~b}$ & $808 \mathrm{~b}$ & $32,6 \mathrm{c}$ \\
\hline H11 (RY1 x TT1) & $28,00 \mathrm{a}$ & $3211 \mathrm{a}$ & $13,00 \mathrm{a}$ & $1374 \mathrm{a}$ & $46,5 \mathrm{~b}$ \\
\hline H21 (RY2 x TT1) & $20,75 \mathrm{~b}$ & $2192 b$ & $9,00 \mathrm{~b}$ & $1027 \mathrm{a}$ & $43,2 \mathrm{~b}$ \\
\hline H31 (RY3 x TT1) & $27,25 \mathrm{a}$ & $2723 \mathrm{a}$ & $9,50 \mathrm{~b}$ & $934 \mathrm{a}$ & $35,1 \mathrm{c}$ \\
\hline H22 (RY2 x TT2) & $19,00 \mathrm{~b}$ & $2091 \mathrm{~b}$ & $10,00 \mathrm{a}$ & $1326 \mathrm{a}$ & $53,5 \mathrm{a}$ \\
\hline H32 (RY3 x TT2) & $18,75 \mathrm{~b}$ & $1915 \mathrm{~b}$ & $6,00 \mathrm{~b}$ & $686 \mathrm{~b}$ & $32,2 \mathrm{c}$ \\
\hline H42 (RY4 x TT2) & $17,50 \mathrm{~b}$ & $1777 \mathrm{~b}$ & $6,25 \mathrm{~b}$ & $691 b$ & $34,7 \mathrm{c}$ \\
\hline H33 (RY3 x TT3) & $20,25 \mathrm{~b}$ & $1935 \mathrm{~b}$ & $8,75 \mathrm{~b}$ & $959 a$ & $43,0 \mathrm{~b}$ \\
\hline H43 (RY4 x TT3) & $25,75 \mathrm{a}$ & $2832 \mathrm{a}$ & $12,00 \mathrm{a}$ & $1316 \mathrm{a}$ & $45,7 \mathrm{~b}$ \\
\hline H53 (RY5 x TT3) & $21,75 \mathrm{~b}$ & $2301 \mathrm{~b}$ & $9,50 \mathrm{~b}$ & $1086 \mathrm{a}$ & $40,9 \mathrm{~b}$ \\
\hline H44 (RY4 x TT4) & $24,50 \mathrm{a}$ & $2535 \mathrm{a}$ & $9,25 \mathrm{~b}$ & $1014 \mathrm{a}$ & $38,1 \mathrm{c}$ \\
\hline H54 (RY5 x TT4) & $27,25 \mathrm{a}$ & 3093 a & $12,00 \mathrm{a}$ & $1311 \mathrm{a}$ & $44,4 \mathrm{~b}$ \\
\hline H64 (RY6 x TT4) & $25,75 \mathrm{a}$ & $2736 \mathrm{a}$ & $9,00 \mathrm{~b}$ & $988 \mathrm{a}$ & $34,6 \mathrm{c}$ \\
\hline H15 (RY1 x TT5) & $27,00 \mathrm{a}$ & $2771 \mathrm{a}$ & $13,25 \mathrm{a}$ & $1497 \mathrm{a}$ & $51,1 \mathrm{a}$ \\
\hline H55 (RY5 x TT5) & $22,75 \mathrm{a}$ & $2314 b$ & $9,25 \mathrm{~b}$ & $1079 \mathrm{a}$ & $41,5 \mathrm{~b}$ \\
\hline H65 (RY6 x TT5) & $21,50 \mathrm{~b}$ & $2310 \mathrm{~b}$ & $11,00 \mathrm{a}$ & $1196 \mathrm{a}$ & $51,8 \mathrm{a}$ \\
\hline Taisho & $20,00 \mathrm{~b}$ & $2109 \mathrm{~b}$ & $8,75 \mathrm{~b}$ & $959 a$ & $45,0 \mathrm{~b}$ \\
\hline Tsuyataro & $25,25 \mathrm{a}$ & $2915 \mathrm{a}$ & $11,25 \mathrm{a}$ & $1342 \mathrm{a}$ & $43,6 b$ \\
\hline Yoshinari & $21,00 \mathrm{~b}$ & $2393 \mathrm{~b}$ & $9,25 \mathrm{~b}$ & $1154 \mathrm{a}$ & $42,1 \mathrm{~b}$ \\
\hline C.V. (\%) & 15,3 & 26,8 & 18,7 & 31,4 & 11,5 \\
\hline
\end{tabular}

Médias seguidas pela mesma letra, nas colunas, não diferem estatisticamente pelo teste de Scott \& Knott (p > 0,05);

RY1 a RY6 = linhagens da população Rensei x Yoshinari; TT1 a TT6 = linhagens da população Tsuyataro x Taisho; H16 a H65= híbridos experimentais. 
Para todas as características de produção avaliadas, observa-se superioridade numérica dos híbridos experimentais com relação a várias linhagens, principalmente da população RY, o que pode ser indício de perda de vigor em função da endogamia nessas linhagens, favorecendo a expressão de heterose nos híbridos, conforme relatado por diversos autores, em estudos sobre pepino (Ghaderi \& Lower, 1978; Filgueira et al., 1986; Lower \& Edwards, 1986; Delaney \& Lower, 1987; Cui et al., 1992; Li et al., 1995; Godoy et al., 2005; Cardoso, 2006). Entretanto, Oviedo et al. (2008) relataram não haver perda de produção com a endogamia em pepino japonês, trabalhando com uma população obtida a partir do híbrido Natsusuzumi.

Estes resultados diferem dos obtidos por Cardoso (2007), pois, para número de frutos total e comercial, este autor observou que todas as linhagens obtidas do híbrido Tsuyataro e algumas oriundas do híbrido Yoshinari apresentaram resultados inferiores aos dos híbridos comerciais. Neste trabalho, algumas linhagens da população TT foram tão produtivas quanto o híbrido comercial Tsuyataro e superiores ao Taisho e Yoshinari. Porém, Cardoso (2007) encontrou híbridos tão produtivos e com mesma produção de frutos comerciais que o melhor híbrido comercial, o que também aconteceu neste trabalho, pois estatisticamente muitos não diferiram do híbrido Tsuyataro, podendo ser destacados os híbridos H16, H26, H11, H43, H54 e H15 e a linhagem TT2, que, para todas as características de produção, permaneceram no grupo superior.

Cardoso (2007) obteve um baixo número de frutos total (híbrido comercial Tsuyataro = 17,20 frutos planta ${ }^{-1}$; híbrido experimental superior $=20,00$ frutos $_{\text {planta }}{ }^{-1}$; linhagem superior $=18,00$ frutos planta ${ }^{-1}$ ), comparado com o deste trabalho (híbrido comercial Tsuyataro $=25,25$ frutos planta $^{-1}$; híbrido experimental superior $=28,00$ frutos planta $^{-1}$; linhagem superior $=25,25$ frutos planta $\left.^{-1}\right)$. Porém, para número de frutos comerciais, Cardoso (2007) obteve valores superiores, provavelmente por causa da maior incidência de oídio nas plantas, que prejudica a área foliar, reduzindo a taxa de frutos comerciais e também pela não retirada precoce dos frutos defeituosos (Nomura \& Cardoso, 2000). No entanto, Cardoso \& Silva (2003), que testaram 19 híbridos comerciais, verificaram que, para o cultivo de verão, o híbrido mais produtivo foi o Tsuyataro (25,4 frutos planta $\left.^{-1}\right)$, assim como foi observado neste experimento.

Para as características de fruto, observou-se que, apesar de se colherem os frutos com um tamanho padrão, houve diferenças significativas para comprimento e diâmetro de fruto, assim como para a relação comprimento/ diâmetro (Tabela 2). Isto deve ter ocorrido por causa das características intrínsecas de cada material. Quanto ao comprimento do fruto, não houve diferença significativa entre os híbridos comerciais, que não diferiram das linhagens TT1, TT5 e TT6, e de 13 dos 18 híbridos experimentais (Tabela 2). Considerando-se que o padrão comercial está em torno de $20 \mathrm{~cm}$ e que a média de todos os tratamentos foi de $21,21 \mathrm{~cm}$, a maioria dos híbridos experimentais está de acordo com o padrão comercial. Porém, Cardoso (2007) observou que o híbrido Yoshinari produziu frutos mais compridos que o híbrido Tsuyataro. O menor comprimento das linhagens da população RY talvez esteja relacionado com o menor comprimento do híbrido Rensei, conforme relatado por Cardoso \& Silva (2003). Considerando-se que este híbrido foi genitor dessas linhagens, a característica de frutos menores deve ter sido herdada pelas linhagens.

Houve diferença estatística entre os genótipos para diâmetro dos frutos. As linhagens RY2 e RY3 foram classificadas como as de maior diâmetro, com valores próximos ao que se deseja comercialmente, pois, para pepino japonês, desejam-se frutos com diâmetro de até $3 \mathrm{~cm}$. Os híbridos comerciais Tsuyataro e Yoshinari não tiveram diferença estatística entre si, mas diferiram do Taisho que apresentou menor diâmetro (Tabela 2).

A relação comprimento/diâmetro dos frutos teve maior variação em relação às demais características (Tabela 2). A linhagem TT1 e o híbrido experimental H31 foram superiores aos demais materiais, inclusive aos híbridos comerciais, que ficaram em uma classificação intermediária, sendo o Taisho aquele com maior valor entre os comerciais. Os materiais de menores médias (RY1, RY2 e RY3) foram os que apresentaram frutos mais curtos e com maior diâmetro.

Quanto à caracterização dos frutos (comprimento, diâmetro e relação comprimento/diâmetro), os híbridos experimentais H16, H22, H33 e H55 foram os mais semelhantes aos híbridos comerciais Tsuyataro e Yoshinari. Desta forma, estes híbridos experimentais, por se assemelharem aos já existentes no mercado, têm um alto potencial de aceitação comercial.

Para a massa média de frutos comerciais, não houve diferença significativa entre os materiais avaliados (Tabela 2). Os valores obtidos foram pouco inferiores aos obtidos por Cardoso \& Silva (2003), para os híbridos Tsuyataro e Yoshinari (149,12 e 155,43 gramas fruto $^{-1}$, respectivamente). Considera-se, porém, como padrão de qualidade, frutos com cerca de $20 \mathrm{~cm}$ de comprimento e $120 \mathrm{~g}$ de massa, semelhantemente ao observado nos materiais avaliados.

Em linhas gerais, foram obtidos híbridos experimentais e linhagens tão produtivos quanto o melhor híbrido comercial avaliado e, às vezes, superiores a dois dos três híbridos comerciais, indicando a superioridade dos materiais avaliados. Estes resultados compro- 
vam a possibilidade de se obterem híbridos tão produtivos quanto os comerciais, com a estratégia de extração de linhagens a partir de híbridos comerciais, conforme relatado por Cardoso (2007). Cramer \& Wehner (1999) também relataram a possibilidade de se obter linhagens tão produtivas quanto os híbridos em pepino, por causa da pequena depressão endogâmica nesta espécie. Além da baixa perda de vigor pela endogamia em algumas linhagens, deve haver predominância de genes com ação aditiva já presentes nos híbridos Taisho ou Tsuyataro, que se manifestaram nessas melhores linhagens, o que não deve ocorrer com os híbridos Rensei e Yoshinari.
Dos 72 valores de características de produção avaliados, dos 18 híbridos experimentais avaliados, 61 tiveram heterose positiva (Tabela 3), o que demonstra superioridade dos híbridos experimentais quando comparados com a média de seus genitores.

Os híbridos H31 (RY3 x TT1) e H11 (RY1 x TT1) destacaram-se com as maiores estimativas de heterose para número de frutos total e massa de frutos total, respectivamente. Já, para número de frutos comerciais e massa de frutos comerciais, o híbrido experimental com maior valor foi o H26 (RY2 x TT6) (Tabela 3).

Os híbridos experimentais H26 e H11 apresentaram médias elevadas para características de produção (núme-

Tabela 2. Médias do comprimento de frutos (COMP), diâmetro de frutos (DIAM), relação comprimento/diâmetro (C/D) e massa média de frutos comerciais (MM)

\begin{tabular}{|c|c|c|c|c|}
\hline \multirow{2}{*}{ Linhagem ou híbrido } & COMP & DIAM & \multirow{2}{*}{ C/D } & \multirow{2}{*}{$\frac{\text { MM }}{\left(\text { g fruto }^{-1}\right)}$} \\
\hline & \multicolumn{2}{|c|}{$(\mathrm{cm})$} & & \\
\hline RY1 & $18,2 \mathrm{c}$ & $2,77 \mathrm{~b}$ & $6,74 \mathrm{e}$ & $115 \mathrm{a}$ \\
\hline RY2 & $18,4 \mathrm{c}$ & $3,13 \mathrm{a}$ & $5,94 \mathrm{e}$ & $113 \mathrm{a}$ \\
\hline RY3 & $20,9 \mathrm{~b}$ & $3,08 \mathrm{a}$ & $6,76 \mathrm{e}$ & $125 \mathrm{a}$ \\
\hline RY4 & $20,8 \mathrm{~b}$ & $2,50 \mathrm{c}$ & $8,29 \mathrm{c}$ & $110 \mathrm{a}$ \\
\hline RY5 & $20,6 b$ & $2,71 \mathrm{~b}$ & $7,61 \mathrm{~d}$ & $116 \mathrm{a}$ \\
\hline RY6 & $20,4 \mathrm{~b}$ & $2,58 \mathrm{c}$ & $7,88 \mathrm{c}$ & $110 \mathrm{a}$ \\
\hline TT1 & $24,1 \mathrm{a}$ & $2,51 \mathrm{c}$ & $9,57 \mathrm{a}$ & $100 \mathrm{a}$ \\
\hline TT2 & $20,4 \mathrm{~b}$ & $2,67 \mathrm{~b}$ & $7,62 \mathrm{~d}$ & $109 \mathrm{a}$ \\
\hline TT3 & $19,5 \mathrm{c}$ & $2,68 \mathrm{~b}$ & $7,30 \mathrm{~d}$ & $110 \mathrm{a}$ \\
\hline TT4 & $20,9 \mathrm{~b}$ & $2,54 \mathrm{c}$ & $8,21 \mathrm{c}$ & $109 \mathrm{a}$ \\
\hline TT5 & $21,7 \mathrm{a}$ & $2,56 \mathrm{c}$ & $8,51 \mathrm{c}$ & $90 \mathrm{a}$ \\
\hline TT6 & $21,9 \mathrm{a}$ & $2,44 \mathrm{c}$ & $8,96 \mathrm{~b}$ & $108 \mathrm{a}$ \\
\hline H16 (RY1 x TT6) & $21,8 \mathrm{a}$ & $2,75 \mathrm{~b}$ & $8,03 \mathrm{c}$ & $113 \mathrm{a}$ \\
\hline H26 (RY2 x TT6) & $20,4 \mathrm{~b}$ & $2,56 \mathrm{c}$ & $7,96 \mathrm{c}$ & $116 \mathrm{a}$ \\
\hline H66 (RY6 x TT6) & $22,4 \mathrm{a}$ & $2,49 c$ & $8,97 \mathrm{~b}$ & $111 \mathrm{a}$ \\
\hline H11 (RY1 x TT1) & $21,5 \mathrm{a}$ & $2,54 \mathrm{c}$ & $8,47 \mathrm{c}$ & $105 \mathrm{a}$ \\
\hline H21 (RY2 x TT1) & $20,9 \mathrm{~b}$ & $2,55 \mathrm{c}$ & $8,23 \mathrm{c}$ & $113 \mathrm{a}$ \\
\hline H31 (RY3 x TT1) & $22,2 \mathrm{a}$ & $2,21 \mathrm{c}$ & $10,03 \mathrm{a}$ & $97 \mathrm{a}$ \\
\hline H22 (RY2 x TT2) & $22,2 \mathrm{a}$ & $2,69 \mathrm{~b}$ & $8,24 \mathrm{c}$ & $132 \mathrm{a}$ \\
\hline H32 (RY3 x TT2) & $21,7 \mathrm{a}$ & $2,44 c$ & $8,89 \mathrm{~b}$ & $111 \mathrm{a}$ \\
\hline H42 (RY4 x TT2) & $22,3 \mathrm{a}$ & $2,52 \mathrm{c}$ & $8,86 \mathrm{~b}$ & $109 \mathrm{a}$ \\
\hline H33 (RY3 x TT3) & $21,7 \mathrm{a}$ & $2,72 \mathrm{~b}$ & $7,97 \mathrm{c}$ & $110 \mathrm{a}$ \\
\hline H43 (RY4 x TT3) & $21,7 \mathrm{a}$ & $2,42 \mathrm{c}$ & $8,98 \mathrm{~b}$ & $113 \mathrm{a}$ \\
\hline H53 (RY5 x TT3) & $19,6 \mathrm{c}$ & $2,27 \mathrm{c}$ & $8,74 \mathrm{~b}$ & $113 \mathrm{a}$ \\
\hline H44 (RY4 x TT4) & $22,2 \mathrm{a}$ & $2,54 \mathrm{c}$ & $8,73 \mathrm{~b}$ & $111 \mathrm{a}$ \\
\hline H54 (RY5 x TT4) & $21,8 \mathrm{a}$ & $2,57 \mathrm{c}$ & $8,52 \mathrm{c}$ & $110 \mathrm{a}$ \\
\hline H64 (RY6 x TT4) & $22,8 \mathrm{a}$ & $2,63 \mathrm{~b}$ & $8,63 \mathrm{~b}$ & $109 \mathrm{a}$ \\
\hline H15 (RY1 x TT5) & $20,4 \mathrm{~b}$ & $2,80 \mathrm{~b}$ & $7,27 \mathrm{~d}$ & $109 \mathrm{a}$ \\
\hline H55 (RY5 x TT5) & $21,2 \mathrm{a}$ & $2,71 \mathrm{~b}$ & $7,86 \mathrm{c}$ & $118 \mathrm{a}$ \\
\hline H65 (RY6 x TT5) & $21,0 \mathrm{~b}$ & $2,64 \mathrm{~b}$ & $8,00 \mathrm{c}$ & $111 \mathrm{a}$ \\
\hline Taisho & $21,5 \mathrm{a}$ & $2,47 \mathrm{c}$ & $8,72 \mathrm{~b}$ & $109 \mathrm{a}$ \\
\hline Tsuyataro & $21,3 \mathrm{a}$ & $2,65 \mathrm{~b}$ & $8,04 \mathrm{c}$ & $119 \mathrm{a}$ \\
\hline Yoshinari & $21,8 \mathrm{a}$ & $2,69 \mathrm{~b}$ & $8,14 \mathrm{c}$ & $121 \mathrm{a}$ \\
\hline C.V. $(\%)$ & 5,3 & 10,5 & 8,1 & 7,2 \\
\hline
\end{tabular}

Médias seguidas pela mesma letra, nas colunas, não diferem estatisticamente pelo teste de Scott \& Knott (p > 0,05);

RY1 a RY6 = linhagens da população Rensei x Yoshinari; TT1 a TT6 = linhagens da população Tsuyataro x Taisho; H16 a H65= híbridos experimentais. 
ro de frutos total e comercial e massa de frutos total e comercial) e também altos valores para heterose. Contudo, nem sempre valores de heterose elevados significam alta produção, pois heterose retrata a superioridade (ou não) do híbrido em relação à média de seus genitores. Hormuzdi \& More (1990) relataram heteroses variando de 4,6 a 247,3\% para produção, em massa, de frutos maduros de pepino por planta. Entretanto, o híbrido com maior heterose $(247,3 \%)$, avaliado por esses autores foi apenas o sétimo mais produtivo entre 24 híbridos avaliados. A heterose foi numericamente elevada pela baixa produção dos genitores. Neste experimento, um dos híbridos com maior heterose para produção de frutos (H26) não foi o mais produtivo, sendo apenas o quinto mais produtivo entre os 18 híbridos experimentais avaliados. Contudo, apresenta-se agrupado, pelo teste de Scott - Knott, junto ao melhor híbrido comercial, Tsuyataro (Tabela 1).

Em média, as estimativas obtidas para heterose foram elevadas, sendo superiores à maioria das relatadas na literatura para pepino. Filgueira et al. (1986) obtiveram heteroses para número de frutos variando de -17,5 a 12,8\% em híbridos de pepino caipira. Já Rubino \& Wehner (1986) relataram heterose para produção total de frutos em pepino para conserva, de $7,4 \%$, em ensaios conduzidos no verão, e de 5,1\%, na primavera. Também Cardoso (2007) obteve heteroses elevadas (-20,3 a 58,8\%) em híbridos experimentais de pepino do tipo japonês, assim como Godoy et al. (2008), em pepino caipira (-5,27 a 36,94\%).
Pela análise da heterose em relação à média dos genitores, pode-se avaliar a importância, ou não, dos efeitos gênicos não aditivos (dominância e epistasia), geralmente associados à condição heterozigota (Vencovsky \& Barriga, 1992). Portanto, para estes híbridos avaliados, os efeitos gênicos não aditivos foram importantes na determinação do potencial produtivo.

Dos 90 valores de heterose em relação à média dos genitores para características de frutos, 60 foram positivos (Tabela 4), sendo mais da metade dos híbridos superiores à média de seus genitores.

As estimativas de heterose em relação à média dos genitores para a característica comprimento de fruto variou de -2,44 a 14,66; para diâmetro de fruto, de -20,93 a 5,56; para a relação comprimento/diâmetro, de -4,65 a 23,64; para a massa média de frutos comerciais, de -13,40 a 19,04 e para percentagem de frutos comerciais, de -27,19 a 40,03 (Tabela 4). Novamente o híbrido experimental H26 (RY2 x TT6) destacou-se por apresentar o maior valor de heterose para a característica percentagem de frutos comerciais.

Todavia, os valores de heterose para as características de frutos foram numericamente inferiores aos observados para as características de produção de frutos. Provavelmente, o ponto de colheita impede a expressão máxima da heterose para características de fruto (comprimento, diâmetro e massa média de frutos) em pepino, pois os frutos foram colhidos com aproximadamente $20 \mathrm{~cm}$, ou seja, muito antes de atingirem o máximo de crescimento.

Tabela 3. Valores de heterose de 18 híbridos experimentais de pepino do tipo japonês em relação à média dos genitores para as características número de frutos total por planta (FT), massa de frutos total por planta (MT), número de frutos comerciais por planta (FC) e massa de frutos comerciais por planta (MC)

\begin{tabular}{|c|c|c|c|c|}
\hline Híbrido & $\begin{array}{c}\text { FT } \\
\left(\mathbf{n}^{0} \text { planta }^{-1}\right)\end{array}$ & $\begin{array}{c}\text { MT } \\
\left(\text { g planta }^{-1}\right)\end{array}$ & $\begin{array}{c}\text { FC } \\
\left(\mathbf{n}^{\mathbf{0}} \text { planta }^{-1}\right)\end{array}$ & $\begin{array}{c}\text { MC } \\
\left(\text { g planta }^{-1}\right)\end{array}$ \\
\hline H16 (RY1 x TT6) & 1,07 & 11,84 & 34,37 & 35,18 \\
\hline H26 (RY2 x TT6) & 31,16 & 19,14 & 95,74 & 97,89 \\
\hline H66 (RY6 x TT6) & 2,27 & 3,61 & 0,00 & 8,20 \\
\hline H11 (RY1 x TT1) & 26,55 & 61,75 & 70,49 & 58,64 \\
\hline H21 (RY2 x TT1) & 14,48 & 12,38 & 63,63 & 70,13 \\
\hline H31 (RY3 x TT1) & 45,33 & 35,39 & 72,72 & 50,03 \\
\hline H22 (RY2 x TT2) & 1,33 & 4,83 & 11,11 & 34,50 \\
\hline H32 (RY3 x TT2) & $-3,22$ & $-6,80$ & $-33,33$ & $-31,66$ \\
\hline H42 (RY4 x TT2) & $-20,90$ & $-18,31$ & $-39,02$ & $-37,31$ \\
\hline H33 (RY3 x TT3) & 7,28 & 2,00 & 2,94 & $-0,33$ \\
\hline H43 (RY4 x TT3) & 19,07 & 40,38 & 23,07 & 24,12 \\
\hline H53 (RY5 x TT3) & 7,4 & 12,85 & $-3,79$ & $-3,07$ \\
\hline H44 (RY4 x TT4) & 14,61 & 24,09 & 12,12 & 14,30 \\
\hline H54 (RY5 x TT4) & 36,25 & 49,86 & 43,28 & 38,32 \\
\hline H64 (RY6 x TT4) & 24,09 & 37,08 & 4,34 & 14,41 \\
\hline H15 (RY1 x TT5) & 22,03 & 44,89 & 63,07 & 60,56 \\
\hline H55 (RY5 x TT5) & 13,04 & 11,25 & 25,42 & 27,35 \\
\hline H65 (RY6 x TT5) & 2,99 & 14,82 & 44,26 & 56,68 \\
\hline
\end{tabular}

RY1 a RY6 = linhagens da população Rensei x Yoshinari; TT1 a TT6 = linhagens da população Tsuyataro x Taisho; H16 a H65= híbridos experimentais.

Rev. Ceres, Viçosa, v. 59, n.4, p. 484-492, jul/ago, 2012 
Tabela 4. Valores de heterose de 18 híbridos experimentais de pepino do tipo japonês em relação à média dos genitores para as características comprimento de frutos (COM), diâmetro de frutos (DIAM), relação comprimento/diâmetro (C/D), massa média de frutos comerciais $(\mathrm{MM})$ e percentagem de frutos comerciais $(\% \mathrm{FC})$

\begin{tabular}{|c|c|c|c|c|c|}
\hline \multirow{2}{*}{ Híbrido } & COM & DIAM & \multirow{2}{*}{ C/D } & \multirow{2}{*}{$\begin{array}{c}\text { MM } \\
\mathbf{g} \\
\end{array}$} & \multirow{2}{*}{$\begin{array}{c}\% \mathrm{FC} \\
\%\end{array}$} \\
\hline & \multicolumn{2}{|c|}{$\mathbf{c m}$} & & & \\
\hline H16 (RY1 x TT6) & 8,81 & 5,56 & 2,29 & 1,61 & 30,23 \\
\hline H26 (RY2 x TT6) & 1,31 & $-8,07$ & 6,84 & 5,27 & 40,03 \\
\hline H66 (RY6 x TT6) & 6,00 & $-0,79$ & 6,53 & 2,02 & $-9,54$ \\
\hline H11 (RY1 x TT1) & 1,75 & $-3,78$ & 3,86 & $-2,26$ & 34,33 \\
\hline H21 (RY2 x TT1) & $-1,43$ & $-9,57$ & 6,12 & 6,15 & 37,77 \\
\hline H31 (RY3 x TT1) & $-1,06$ & $-20,93$ & 22,84 & $-13,40$ & 15,29 \\
\hline H22 (RY2 x TT2) & 14,66 & $-7,24$ & 21,53 & 19,04 & 18,42 \\
\hline H32 (RY3 x TT2) & 5,12 & $-15,13$ & 23,64 & $-5,19$ & $-27,19$ \\
\hline H42 (RY4 x TT2) & 8,51 & $-2,51$ & 11,37 & 0,07 & $-23,39$ \\
\hline H33 (RY3 x TT3) & 7,41 & $-5,39$ & 13,37 & $-6,50$ & 0,34 \\
\hline H43 (RY4 x TT3) & 7,90 & $-6,38$ & 15,20 & 2,37 & 4,40 \\
\hline H53 (RY5 x TT3) & $-2,44$ & $-15,61$ & 17,23 & $-0,44$ & $-13,15$ \\
\hline H44 (RY4 x TT4) & 6,50 & 0,79 & 5,81 & 0,98 & 2,55 \\
\hline H54 (RY5 x TT4) & 5,08 & $-2,09$ & 7,71 & $-2,13$ & 9,85 \\
\hline H64 (RY6 x TT4) & 10,27 & 2,73 & 7,27 & $-0,56$ & $-15,78$ \\
\hline H15 (RY1 x TT5) & 2,23 & 5,06 & $-4,65$ & 6,50 & 36,69 \\
\hline H55 (RY5 x TT5) & 0,28 & 2,84 & $-2,48$ & 15,38 & 10,98 \\
\hline H65 (RY6 x TT5) & 0,16 & 2,72 & $-2,37$ & 11,85 & 35,83 \\
\hline
\end{tabular}

RY1 a RY6 = linhagens da população Rensei x Yoshinari; TT1 a TT6 = linhagens da população Tsuyataro x Taisho; H16 a H65= híbridos experimentais.

\section{CONCLUSÕES}

Foram obtidos híbridos experimentais tão produtivos quanto os híbridos comerciais. A maioria dos híbridos é superior às linhagens, mostrando a importância da heterose para produção de frutos.

\section{AGRADECIMENTOS}

Os autores agradecem à FAPESP (Processo $n^{\circ}$ 06/ 57674-0), pela concessão da bolsa de mestrado e pelo auxílio à pesquisa (Processo 07/56122-6).

\section{REFERÊNCIAS}

Cañizares KAL (1998) A cultura de pepino. In: Goto R \& Tivelli SW (Eds.) Produção de hortaliças em ambiente protegido: condições subtropicais. São Paulo, Fundação Editora da UNESP. p. 195-223.

Cardoso AII (2002) Avaliação de cultivares de pepino tipo caipira sob ambiente protegido em duas épocas de semeadura. Bragantia, 61:43-48.

Cardoso AII (2006) Dialelo entre híbridos de uma população de pepino caipira. Horticultura Brasileira, 24:259-263.

Cardoso AII (2007) Avaliação de linhagens e híbridos experimentais de pepino do grupo varietal japonês sob ambiente protegido. Bragantia, 66:469-475.

Cardoso AII \& Silva N (2003) Avaliação de híbridos de pepino tipo japonês sob ambiente protegido em duas épocas de cultivo. Horticultura Brasileira, 21:170-175.

Cardoso AII \& Wilcken SRS (2008) Nematóides assustam produtores de tomate e pepino. Campo e Negócio HF, 34:38-39.
Cramer CS \& Wehner TC (1999) Little heterosis for yield and yield components in hybrids of six cucumber inbreds. Euphytica, 110:99-108.

Cruz CD \& Regazzi A J (2004) Modelos biométricos aplicados ao melhoramento genético. 2a ed. Viçosa, Editora UFV. 390p.

Cui HW, Qi YT, Liu JH \& Ren ZB (1992) Correlation between parents and F1 progeny in earliness heterosis and the estimation of traits limits of parents. Report Cucurbit Genetics Cooperative, $15: 13-16$.

Delaney DE \& Lower RL (1987) Generation means analysis of plant characters in crosses between two determinate cucumber lines and Cucumis sativus var. hardwickii. Journal of the American Society for Horticultural Science, 112:707-711.

Filgueira FAR (2003) Novo manual de olericultura: agrotecnologia moderna na produção e comercialização de hortaliças. Viçosa, Universidade Federal de Viçosa. 402p.

Filgueira FAR, Giordano LB, Ferreira PE \& Vecchia PTD (1986) Avaliação de híbridos F1 de pepino do tipo caipira. Horticultura Brasileira, 4:17-20.

Ghaderi A \& Lower RL (1978) Heterosis and phenotypic stability of F1 hybrids in cucumber under controlled environment. Journal of the American Society for Horticultural Science, 103:275-278.

Godoy AR, Oviedo VRS \& Cardoso AII (2005) Análise endogâmica de uma população de pepino caipira. Horticultura Brasileira, 23:785-788.

Godoy AR, Higuti ARO \& Cardoso AII (2008) Produção e heterose em cruzamentos entre linhagens de pepino do grupo caipira. Bragantia, 67:817-825.

Hormuzdi SG \& More TA (1990) Heterosis studies in cucumber. Indian Journal of Horticulture, 46:73-79.

Li JW, Li JW \& Wei ZD (1995) Genetic analysis for major agronomic characters in cucumber (Cucumis sativus L.). Acta Horticulturae, 402:388-391. 
Lower RL \& Edwards MD (1986) Cucumber breeding. In: Basset MJ (Ed.) Breeding vegetable crops. Westport, Avi Publishing. p. 173-207.

Machado CA, Rodrigues CDS, Weirich M \& Chagas PRR (2001) Avaliação de produção e de resistência ao oídio de híbridos de pepino cultivado no sistema de agricultura natural protegido. Horticultura Brasileira, 19:241.

Maluf WR (2001) Heterose e emprego de híbridos $F_{1}$ em hortaliças. In: Nass LL (Ed.) Recursos genéticos e melhoramentoplantas. Rondonópolis, Fundação MT. p.237-356.

Miranda Filho JB \& Gorgulho EP (2001) Cruzamentos com testadores e dialelos. In: NASS LL (Ed.) Recursos genéticos e melhoramento- plantas. Rondonópolis, Fundação MT. p.650-671.

Nomura ES \& Cardoso AII (2000) Redução da área foliar e o rendimento do pepino japonês. Scientia Agricola, 57:257-261.
Oviedo VRS, Godoy AR \& Cardoso AII (2008) Imbreeding Performance of advanced generation from a hybrid Japanese cucumber. Scientia Agricola, 65:553-556.

Robinson RW \& Decker-Walters DS (1999) Cucurbits. Cambridge, CAB International. 226p.

Rubino DB \& Wehner TC (1986) Effect of inbreeding on horticultural performance of lines developed from an openpollinated pickling cucumber population. Euphytica, 35:459464.

Vencovsky R \& Barriga P (1992) Genética biométrica no fitomelhoramento. Ribeirão Preto, Revista Brasileira de Genética. 496 p. 